\title{
La garantía de los DESCA a través del diálogo judicial y arbitral
}

Fecha de recepción: 24 de septiembre de 2018

Fecha de aprobación: 17 de noviembre de 2019

Doi: https://doi.org/10.12804/revistas.urosario.edu.co/acdi/a.8672

\section{Juan Felipe Solórzano Quintero*}

Resumen: el escrito muestra, en primer lugar, la importancia de los derechos sociales, económicos, culturales y ambientales. En segundo lugar, refleja que en la actualidad estos no se encuentran protegidos del todo, en especial en el ámbito del derecho internacional de inversiones. De esta forma los laudos producto de las disputas entre Estados e inversores pueden afectar la instrumentalización de los derechos sociales, ya que restringirían de manera drástica potestades al Estado. En consecuencia, se hace necesario que los árbitros se sometan a los derechos humanos. Para ello, en el Max Planck de derecho público comparado y de derecho internacional se ha desarrollado una teoría conocida como el ius constitutionale commune en América Latina, que permitiría someter a los árbitros no solo a los derechos de las personas, sino que también podría tener en cuenta las decisiones de los tribunales encargados de proteger los derechos humanos v.gr. la Corte Interamericana de Derechos Humanos.

* Abogado con máster en derecho administrativo de la Universidad Colegio Mayor de Nuestra Señora del Rosario y doctor por la Universidad de Salamanca. Miembro de la red Alumni del Max-Planck-Institut für ausländisches öffentliches Recht und Völkerrecht. ORCID 0000-0002-5260-6464 Correos electrónicos: juansolorzano@usal.es y juanfelipe06@ hotmail.com

Para citar este artículo: Solórzano, Juan Felipe. "La garantía de los DESCA a través del diálogo judicial y arbitral”. Anuario Colombiano de Derecho Internacional (ACDI) 13, (2020): 133-164. https://doi.org/10.12804/ revistas.urosario.edu.co/acdi/a.8672 
Palabras clave: ius constitutionale commmune, tribunales de arbitramento, tratados bilaterales de protección de inversiones, derechos sociales, cortes nacionales.

\title{
The Guarantee of DESCA Through Judicial and Arbitration Dialogue
}

\begin{abstract}
This paper illustrates the importance of social, economic, and cultural rights and shows that these rights are not completely protected, especially in international investment law. Thus, the judgments resulting from the disputes between the States and investors can affect the realization of social rights, since authorities can drastically restrict the power of the State. Therefore, it is necessary that the arbitrators submit to human rights. As a result, at the Max Planck Institute for Comparative Public Law and International Law, a constitutional theory known as Ius Constitutionale Commune in Latin America was developed, which allows arbitrators so submit to human rights, as well as to make decisions based on human rights courts v. g. Inter-American court of Human Rights.
\end{abstract}

Keywords: Ius constitutionale commmune, arbitrations courts, bilateral investment trades, social rights, national courts.

A garantia dos DESCA através do diálogo judicial e arbitral

Resumo: o escrito mostra. Em primeiro lugar, a importância dos direitos sociais, econômicos, culturais e ambientais. Em segundo lugar, reflete que na atualidade não todos encontram-se protegidos, especialmente no âmbito do direito internacional de investimentos. Desta forma, as sentenças produto das disputas entre Estados e investidores podem afetar a instrumentalização dos direitos sociais, pois podem restringi-lhe de maneira drástica potestades ao Estado. Por consequência, se faz necessário que os árbitros se submetam aos direitos humanos. Para isto, no Max Planck de Direito público comparada e de Direito Internacional se tem desenvolvido uma teoria conhecida com o ius constitutionale commune na América Latina que permitiria submeter aos árbitros não só aos direitos das pessoas, mas que também poderiam ter em conta as decisões dos tribunais 
encarregados de proteger Direitos Humanos v.gr. Corte Interamericana de Direitos Humanos.

Palavras-chave: ius constitutionale commmune, tribunais de arbitramento, tratados bilaterais de proteção de investimentos, direitos sociais, cortes nacionais.

\section{Introducción}

El presente trabajo pretende mostrar a partir del análisis de la teoría del ius constitutionale commune y de la importancia de los derechos económicos, sociales culturales y ambientales la necesidad improrrogable que los árbitros en materia de inversiones extranjeras generen un diálogo entre las diferentes cortes nacionales e internacionales, a fin de generar un sistema jurídico ordenado y coherente, que garantice la preponderancia de la persona, más allá del beneficio de los intereses del mercado. Esta premisa parte del hecho de que los tribunales de arbitramento interfieren de manera directa en asuntos que atañen al derecho público, como por ejemplo la toma de decisiones públicas o incluso la materialización de los derechos de los ciudadanos, por lo que sus decisiones tienen que adecuarse a los postulados del derecho constitucional.

De esta manera se iniciará con el análisis de los derechos sociales, económicos y culturales que permiten al hombre tener unas condiciones mínimas de vida, que son un medio para garantizar la dignidad humana. ${ }^{1}$ Pese a su importancia y relevancia en la actualidad, nos enfrentamos al desmantelamiento de estos derechos. En primer lugar, por la dificultad de justiciabilidad que en ocasiones presentan. En segundo, por su carácter programático, el cual ha servido de excusa para restarles importancia. En tercero, por las transformaciones del modelo de Estado y la concepción del Estado-nación, los cuales se han visto enfrentados a movimientos como la globalización, las pandemias, las inmigraciones y el protagonismo de la economía que han cuestionado su papel como garante de los derechos. ${ }^{2}$

\footnotetext{
1 Eusebio Fernández García, Dignidad bumana y ciudadanía cosmopolita, (Madrid: Dykinson, 2001).

2 Gerardo Pisarello Prados, "Los derechos sociales y sus 'enemigos': Elementos para una reconstrucción garantista," En Defender y repensar los derechos sociales en tiempo de crisis, editado por Gerardo Pisarello Prados, (Barcelona: Observatorio DESC, 2009), 13-22.
} 
En este orden de ideas, en la actualidad, el Estado ha dejado de ser el único actor en la protección de los derechos de las personas. En consecuencia, han entrado a ser parte organizaciones internacionales y supranacionales, tribunales internacionales y ONG que propenden por el cumplimiento de los Estados y demás autoridades públicas de todos los postulados constitucionales y las obligaciones internacionales en materia de derechos humanos. ${ }^{3}$ Pese a lo anterior y al sistema que se ha generado a nivel internacional, no se puede someter a todas las autoridades a estos derechos. Se encuentra que, en materia de derecho internacional, la primacía de la cual goza la persona no es respetada por todos los organismos internacionales. En materia de derecho internacional público económico, particularmente el de inversiones, cada día se encuentra en un aparente enfrentamiento con los derechos de los individuos, en especial los derechos sociales, económicos y culturales.

En la segunda parte del trabajo se estudiará cómo los tribunales de arbitramento de inversiones en ocasiones pueden tomar decisiones que afectan la materialización de los derechos sociales y del modelo de Estado social y democrático de derecho, y la toma de decisiones públicas. ${ }^{4}$ De esta manera se verá cómo a partir de la teoría del ius constitutionale commune se puede generar un diálogo armónico entre cortes, inclusive contando con los tribunales de arbitramento, con el fin de propender por la garantía de los derechos de las personas. De igual forma, se concluye que es necesario repensar esta rama del derecho en la medida que los árbitros, a través de sus laudos, evalúan el quehacer del Estado, asemejándose a los jueces administrativos. Esta es una razón más, a parte de la necesidad de

3 Respecto de las transformaciones del Estado-nación véase Jessica Mathews, "Power Shift," Foreign Affairs 79, (1997); José María Serna de la Garza, Globalización gobernanza: las transformaciones del Estado y sus implicaciones para el derecho público: contribución para una interpretación del caso guardería ABC, (México, UNAM, 2010).

4 Gerardo Pisarello Prados, "Globalización, mundialización y crisis del concepto de soberanía", en Globalización, mundialización y crisis del concepto de soberania: algunos afectos en América Latina y en Europa editado por Gerardo Pisarello Prados, (Alicante: Ediciones Universidad de Alicante, 2000), 25 ss; Pisarello Prados, "Los Derechos sociales y sus 'enemigos'..., 13-22; Juan Bohoslavsky y Justo Bautista, "Compatibilizando derechos de los inversores extranjeros y derechos humanos: ¿Por Qué? ¿Cómo? ¿Quién? ¿Cuándo?,” en International Investment Law in Latin America: Problems and Prospects editado por Atilla Tanzi et al., (Holanda, Nijhoff international investment law series, 2016), 681-98; Juan Bohoslavsky y Justo Bautista, Protección del derecho humano al agua y arbitrajes de inversión, (Santiago de Chile: CEPAL, 2011). 
generar un sistema jurídico protector del individuo, para que el arbitraje de inversiones incluya las normas en materia de derechos de las personas dentro de sus casos.

\section{Metodología}

El presente escrito es el resultado de una investigación realizada en varias etapas en el Instituto Max-Planck de derecho público comparado y derecho internacional. En este proceso investigativo se estudió el ius constitutionale commune en Latinoamérica, teoría que pretende materializar la garantía de los derechos de las personas por parte de los Estados.

En este sentido y visibilizando los inconvenientes que se presentan en justiciabilidad y aplicabilidad de los derechos económicos, sociales, culturales y ambientales (DESCA) en materia de inversiones, consideramos necesario evaluar la posibilidad de aplicación del ius constitutionale commune en este ámbito del derecho. Lo anterior, con el fin de advertir, en primer lugar, la necesidad apremiante de someter a los tribunales de arbitramento a los DESCA y, en segundo lugar, la de establecer una medida correctiva, particularmente el dialogo entre cortes, que jurídicamente puede tener aplicabilidad en el derecho referente a las inversiones producto de tratados bilaterales de inversiones que se verá reflejado en una mejor materialización de los mencionados derechos.

En este sentido, el trabajo tiene como principal objetivo el estudio de conceptos jurídicos que permitirán establecer un estado del arte en materia de DESCA, la teoría del ius constitutionale commune y los principios básicos del derecho internacional económico público referidos al tema de inversiones. Los anteriores conceptos permitirán desde una visión normativa establecer la posibilidad existente de determinar si los árbitros deben someterse a los DESCA.

De esta suerte, el trabajo se encuadra dentro de una metodología deductiva por cuanto parte de nociones generales que permiten llegar al análisis de un tema determinado que es el diálogo que debe existir entre jueces y árbitros en materia de garantía de derechos. Para ello, se sigue un método de investigación documental, ${ }^{5}$ que lleva a concluir que este escrito es de revisión bibliográfica y conceptual.

\footnotetext{
5 Nancy Solano y Myriam Sepúlveda, Metodología de la investigación social jurídica, (Bogotá, Ibáñez, 2008).
} 


\section{La necesidad de tomar en serio los DESCA}

En nuestros días, es común hacer alusión a los DESCA como una de las grandes conquistas del siglo pasado. Incluso, en muchas de las constituciones occidentales se tipifican estos derechos y, como lo reconoce Aguilar Cavallo, ${ }^{6}$ en la región latinoamericana, lugar donde existe una gran brecha entre la implementación y el goce efectivo de estos, se han incorporado diferentes mecanismos jurisdiccionales para su protección dirigidos a mejorar la situación antes descrita — véase por ejemplo la Convención Americana sobre Derechos Humanos, el protocolo adicional a la Convención Americana sobre Derechos Económicos Sociales y Culturales, y el Pacto Internacional de Derechos Económicos, Sociales y Culturales, entre otros-. De la misma manera, al otro lado del atlántico, se observa cómo la Unión Europea (UE) ha trazado ciertos parámetros en materia de protección de los DESCA. Así cuenta, entre otros, con una política social dentro del Tratado de Funcionamiento de la Unión Europea, la Carta Social Europea de 1961 y la Carta de Derechos Fundamentales de 2000 que claramente los perpetúan y deben ser garantizados por ella y cada uno de los Estados miembros.

Pese a su importancia en el mundo actual, estos derechos en términos de exigibilidad y de justiciabilidad están por debajo de los conocidos derechos civiles y políticos. ${ }^{7} \mathrm{Al}$ respecto, es sabido que los DESCA están asentados en valores diferentes de los demás derechos, motivo por el cual se menosprecia su justiciabilidad. En este sentido, los derechos civiles y políticos en principio se relacionan con valores como la libertad y la dignidad, mientras que los DESCA "tendrían un vínculo remoto con dichos principios y valores. Estarían, más bien, ligados a otros diferentes como la igualdad material o la solidaridad, cuya satisfacción solo residiría justificada una vez aseguradas debidamente la libertad o la dignidad". ${ }^{8}$

\footnotetext{
6 Gonzallo Aguilar Cavallo, "¿Son los derechos sociales solo apreciaciones? Perspectivas de la Comisión Interamericana de Derechos Humanos", en Construcción y papel de los derechos sociales fundamentales editado por Armin von Bogdandy, (México, Instituto de Investigaciones Jurídicas, 2011), 197-234.

7 Pisarello Prados, “Los Derechos sociales y sus 'enemigos'...

8 Ernst Forsthoff, "Concepto y esencia del Estado Social de Derecho", en El estado social, editado por Wolfgang Abendrot Ernst Forsthoff y Karl Doehring, (Madrid: Centro de Estudios Constitucionales, 1986), 99-101.
} 
Pisarello Prados ${ }^{9}$ y Aguilar Cavallo ${ }^{10}$ ponen de presente un asunto que se encuentra desde la inclusión de la cláusula social en el modelo de Estado: el carácter prestacional de los DESCA. Es menester decir que cuando estos derechos cobraron carta de naturaleza, se empezó a discutir de manera vehemente sobre su contenido indeterminado y la necesidad de intervención asidua por parte de gobiernos y parlamentos. ${ }^{11}$ Esto repercute en su justiciabilidad, en primer lugar, porque existen constituciones, como la española, que tratan de manera diferente a los DESCA. En segundo lugar, y como lo reconoce Arango, ${ }^{12}$ existe una indeterminación en quién es el obligado en la prestación del derecho: su familia, el Estado o la comunidad internacional. Lo anterior lleva, según el autor, a un problema concerniente a que si no existe una prescripción legal que determine los obligados y sus deberes respecto de la materialización del derecho, no se puede establecer a ciencia cierta cuándo se está en presencia de la conculcación de un DESCA y en consecuencia no se puede establecer un método que permita saber objetiva y razonablemente el quebrantamiento de determinado derecho.

Ahora bien, pese a la indeterminación que pueden tener los DESCA, por ser considerados prestacionales, se puede decir que una visión restrictiva y literal resulta errónea. Si nos detenemos a observar los derechos civiles y políticos, estos también requieren prestaciones positivas y negativas. En este orden de cosas, para garantizar derechos clásicos como la libertad, la propiedad o el sufragio se requieren acciones por parte del Estado que implican recursos económicos y la iniciativa gubernamental y parlamentaria. ${ }^{13}$

\footnotetext{
9 Pisarello Prados, "Los Derechos sociales y sus 'enemigos'...

10 Aguilar Cavallo, “¿Son los derechos sociales solo apreciaciones..., 197-234?

11 Forsthoff, "Concepto y esencia del Estado Social...

12 Rodolfo Arango, El concepto de los derechos, (Bogota: Legis, 2015), 7-120.

13 Cécile Fabre, Social Rights under the Constitution, (Oxford, Oxford University Press, 2000); Óscar Parra Vera, "La justiciabilidad de los derechos económicos sociales y culturales en el sistema interamericano a la luz del artículo 26 de la Convención Americana: El sentido y la promesa del caso Lagos del Campo", en Inclusión, ius commune y justiciabilidad de los DESC en la jurisprudencia interamericana: el caso Lagos del Campo y los nuevos desafíos editado por Eduardo Ferrer Mac-Gregor et al., (Querétaro: Instituto de Estudios Constitucionales del Estado de Querétaro, 2018); Flavia Piovesan, "Protección de los derechos sociales: reto de un ius commune para Sudamérica, en Construcción y papel de los derechos sociales fundamentales
} 
Al respecto, la Corte Constitucional de Sudáfrica recuerda en el caso Government of the Republic of South Africa. \& Ors v. Grootboom \& Ors que:

These rights are, at least to some extent, justiciable. (...), many of the civil and political rights entrenched in the constitutional text before this Court for certification in that case, will give rise to similar budgetary implications without compromising their justiciability. The fact that socio-economic rights will almost inevitably give rise to such implications does not seem to us to be a bar to their justiciability. At the very minimum, socio-economic rights can be negatively protected from improper invasion. ${ }^{14}$

El anterior aparte resulta importante para demostrar que la obligatoriedad de los derechos es un tema que va más allá de las obligaciones presupuestarias demandadas al Estado. De esta forma, los derechos son una cuestión allende de su clasificación y consideramos deberían centrarse en su finalidad, que es la protección de la persona. Pensamos que los DESCA se pueden entender mejor desde la siguiente construcción. Para obtener una vida decente, bienestar general y autonomía, los valores que se relacionan directamente con los derechos civiles y políticos, no son suficientes. Es necesario entonces contar también con ciertos aspectos de los derechos sociales, ya que estos permiten concretar gran parte los tradicionales derechos civiles y políticos. Si se garantizan y se satisfacen las necesidades de los seres humanos, se garantiza también un mejor nivel de autonomía y bienestar. ${ }^{15}$ Es de resaltar cómo la Corte Interamericana de Derechos Humanos (CIDH) ha manifestado el carácter de indivisibilidad e interdependencia de los derechos civiles y políticos con los DESCA. Así, en la sentencia del 21 de mayo de 2013 , la $\mathrm{CIDH}^{16}$ recordó que los derechos deben entenderse sin ninguna jerarquía entre sí y son exigibles a todas

editado por Armin von Bogdandy et al., (México: Universidad Autónoma de México), 339-380.

14 Sudáfrica, Corte Constitucional, Government of the Republic of South Africa. \& Ors v Grootboom \& Ors, 17 de diciembre de 1999.

15 Fabre, Social Rights under...

16 Corte Interamericana de Derechos Humanos, sentencia caso Suárez Peralta c. Ecuador, 21 de mayo de 2013. 
las autoridades que estén obligadas a su protección. ${ }^{17}$ En consecuencia, la atención y el grado de protección de los DESCA debe ser la misma que tienen los derechos civiles y políticos. ${ }^{18}$

Al respecto, la Declaración y Programa de Acción de Viena sostiene, en el apartado I. 5, que los derechos humanos son interdependientes y están relacionados entre ellos. De la misma manera, recuerda que tanto los Estados como la comunidad internacional están obligados a su guarda y materialización. Lo anterior, refuerza la idea que los DESCA deben encontrarse a la par de los derechos civiles y políticos independientemente del elemento prestacional que tienen per se. ${ }^{19}$

Ahora bien, para reforzar esta idea se pueden revisar las aportaciones doctrinarias desarrolladas en Alemania, ${ }^{20}$ donde se encuentra la teoría de la doble vertiente de los derechos fundamentales (Der Doppelcharakter der Grundrechte), que se refiere a que los derechos no tienen únicamente un carácter subjetivo o de defensa de la persona frente a las actuaciones del Estado, sino que también generan un orden que delimita de manera objetiva el quehacer de las autoridades públicas. ${ }^{21}$ Esto implica que devienen en normas objetivas de principio y decisiones axiológicas. Al respecto Hesse sostiene que los derechos, al tener un doble carácter, aseguran la libertad del individuo frente a intromisiones de los poderes públicos y se fundan como principios axiológicos, objetivos y robustecidos de fuerza normativa. ${ }^{22}$ Lo anterior, lleva a pensar que todas las autoridades públicas deben tener en cuenta a la persona, ya que los derechos demarcarán el devenir de su quehacer, y no se debe perder de vista que son estos los

17 Corte Interamericana de Derechos Humanos, sentencia caso Suárez..., en especial voto recurrente del juez Ferrer- Mac-Gregor.

18 Miguel Carbonell, y Eduardo Ferrer Mac-Gregor, Los derechos sociales y su justiciabilidad directa, (México, Universidad Autónoma de México, 2014).

19 Corte Interamericana de Derechos Humanos, sentencia caso Acevedo Buendía c. Perú, 1 de julio de 2009.

20 Wolfgang Böckenförde, Escritos sobre derechos fundamentales, (Baden-Baden: Nomos, 1993).

21 Marta León Alonso, La protección constitucional de la salud en el marco del Estado Social y Democrático de Derecho, (Salamanca: Ediciones Universidad de Salamanca, 2009).

22 Konrad Hesse, Grun₹üge des Verfassungsrecbts der Bundesrepublik. Deutschland, (Heidelberg: C.F. Müller, 1991); Konrad Hesse, "El significado de los derechos fundamentales", en Manual de derecho constitucional editado por Antonio López Pina, (Madrid: Marcial Pons, 2001), 90-91. 
que permitirán que la dignidad de la persona como valor supremo pueda materializarse efectivamente. ${ }^{23}$

Ahora bien, si se revisa la teoría de los derechos fundamentales de Alexy, ${ }^{24}$ se encuentra que las normas de derecho fundamental pueden ser reglas, pero también mandatos de optimización. ${ }^{25}$ Este punto sirve de elemento clave para dar cuenta de que los derechos fundamentales no solo se imponen como normas de abstención o limite, sino que además devienen en principios que irradian el ordenamiento jurídico y vinculan a las autoridades públicas en su consecución.

Esto significa que los derechos fundamentales son derecho aplicable directamente y dejan de ser vistos como disposiciones programáticas. Por lo tanto, vinculan a los poderes estatales y a los particulares en su cumplimiento. ${ }^{26}$ Esto lleva a concluir que el contenido axiológico requiere un permanente desarrollo de su contenido normativo y que los poderes públicos deben tener un papel proactivo en la protección de los derechos. ${ }^{27}$

Respecto a lo anterior, se evidencia la obligatoriedad de los derechos fundamentales por parte de los poderes públicos. En este punto se puede generar el cuestionamiento de la inclusión de los DESCA y su consideración en la categoría de fundamentales. Se piensa que el carácter objetivo de los derechos fundamentales proviene de la consolidación del Estado social. $\mathrm{Al}$ respecto León sostiene que para

el disfrute de los derechos y libertades exige una serie de condiciones materiales, surge el status positivus socialis que incluye los denominados

23 Hesse, "El significado de los derechos..., 90-91.

24 Robert Alexy, Teoría de los derechos fundamentales, (Madrid: Centro de Estudios Políticos y Constitucionales, 2007).

25 Carlos Bernal Pulido, "Capítulo introductorio", en Teoría de los derechos fundamentales editado por Robert Alexy, (Madrid: Centro de estudios políticos y constitucionales, 2007): xxv y ss.

26 Böckenförde, Escritos sobre derechos...

27 León Alonso, La protección constitucional de la salud...

Respecto de la incorporación de la concepción objetiva en la jurisprudencia española pueden verse como ejemplos los pronunciamientos proferidos por el Tribunal Constitucional, particularmente STC 53/1985, de 11 de abril, fundamento jurídico 4 y STC 25/1981, de 14 de julio, fundamento jurídico 5. Un ejemplo en Latinoamérica puede ser el colombiano. Al respecto puede verse, Corte Constitucional, Sentencia C-587 de 1992 del 12 de noviembre y Sentencia T- 596 de 1992 del 10 de diciembre. 
derechos económicos, sociales y culturales. Estos derechos garantizan el pleno desarrollo de la subjetividad humana y, por tanto, pasan a integrar la categoría de los derechos fundamentales. ${ }^{28}$

Si bien esta teoría se generó dentro de un ámbito estatal, cabe decir, como lo sostiene Böckenförde, ${ }^{29}$ que el contenido axiológico que se encuentra en el carácter subjetivo de los derechos irradia todos los ámbitos del derecho. Es por lo que esta teoría trasciende el espacio del contexto de Estado-nación y repercute en el derecho internacional.

Una forma de justificar la premisa anterior es la estatalidad abierta (die offene Staatlichkeit). ${ }^{30}$ Esta teoría afirma que existe una permeabilidad en el ordenamiento nacional, que cada vez está cobrando más vigencia en las constituciones occidentales. De esta manera, se puede predicar una cooperación internacional en los planos regional e internacional y un mayor sometimiento por parte del Estado a la dignidad y a los derechos humanos, ya que se inserta de una manera más directa con las obligaciones internacionales que emanan del ordenamiento jurídico internacional. De esta manera "el Estado constitucional contemporáneo solo puede entenderse como un Estado situado internacionalmente y, por lo tanto, limitado en idéntica perspectiva, particularmente en materia de derechos humanos". ${ }^{31}$

Entonces, si se parte de la existencia de una cooperación entre dos sistemas jurídicos y una exaltación del deber del Estado de proteger los derechos humanos, resultaría también lógico pensar que la teoría del doble carácter de los derechos fundamentales se puede transpolar, toda vez que una de las razones de la génesis del derecho internacional fue la de proteger los derechos de las personas, en especial de aquellas que se encuentran en un grado de vulnerabilidad latente. ${ }^{32}$ Por lo tanto, las

\footnotetext{
28 Ibíd., 326.

29 Böckenförde, Escritos sobre derechos...

30 Este término fue acuñado por primera vez por Klaus Vogel, en Die Verfassungentscheidung des Grungesetzes für die internationale Zusammenarbeit.

31 Mariela Morales Antoniazzi, "El nuevo paradigma de la apertura de los órdenes constitucionales: una perspectiva sudamericana”, en Soberanía y Estado abierto en América Latina y Europa editado por Armin von Bogdandy et al., (México: Instituto de Investigaciones Jurídicas, 2014), 270.

32 Armin von Bogdandy, "La protección de los vulnerables: un ejemplo de gobernanza
} 
organizaciones de carácter internacional deberán tener en cuenta desde una visión objetiva los derechos de las personas.

Así las cosas, vista la obligatoriedad que existe por parte de las instituciones públicas nacionales e internacionales de tener en cuenta en su quehacer los derechos fundamentales, resulta cierto que el debate generado respecto de la 'inferioridad' de los DESCA es a todas luces un mecanismo tendiente a priorizar otros intereses diferentes a la dignidad de la persona, ya que como mencionamos anteriormente, el conjunto de los derechos permite que exista la posibilidad en una comunidad de materializar un bienestar general.

Por otra parte, teniendo en cuenta la importancia de los derechos sociales en la región latinoamericana, se habla de un nuevo constitucionalismo que tiene como una de sus banderas la salvaguarda de los DESCA. De igual manera, se hace un especial énfasis en la consolidación de los postulados participativos, el reconocimiento de una diversidad social (pueblos indígenas, comunidades afros, etc.) y la garantía de un Estado material de derecho que brinden garantías a las culturas y comunidades que conviven en un espacio. Finalmente, se debe hacer énfasis en la necesidad de intervención del Estado en la economía para hacerles frente a las políticas neoliberales propias de la apertura descontrolada de los mercados nacionales. ${ }^{33}$

Luego de haber determinado la relevancia de los DESCA, es claro que estos permiten la materialización de los derechos civiles. Por lo estudiado, se podría afirmar que en su conjunto son parte determinante de la materialización de la dignidad de la persona. En este entendido y teniendo en cuenta su relevancia resultaría evidente que este tipo de derechos fuese salvaguardado en todas las situaciones en las que se vieran amenazados. Incluso, se creería que el desarrollo normativo internacional, incluyendo el económico, permitiría afirmar que los DESCA son un pilar en el ejercicio de las relaciones jurídicas. Lo anterior, teniendo en cuenta que los tratados internacionales de derecho público son interpretados sistemáticamente en virtud del artículo 31 de la Convención de Viena.

posnacional", en Construcción y papel de los derechos sociales fundamentales editado por Armin von Bogdandy et al., (México: Instituto de Investigaciones Jurídicas, 2011).

33 Armin von Bogdandy et al., "Ius constitutionalle commune en América Latina: un enfoque regional del constitucionalismo transformador', en Ius constitutionale commune en América Latina: textos básicos para su comprensión editado por Armin von Bogdandy et al., (Querétaro: Instituto de Estudios Constitucionales del Estado de Querétaro, 2017), 17-54. 
Esto significaría que el derecho internacional público de inversiones debe tener en cuenta los derechos de las personas: i) Existe la sistematicidad en materia de interpretación de tratados; ii) Los tribunales de arbitramento tienen competencia en los tratados internacionales firmados entre dos o más Estados; iii) Una de las partes que va a concurrir ante este tipo de litigios es un Estado, y iv) la fuente de su competencia es el derecho público.

\section{E1 ius constitutionale commune y el dialogo con los árbitros en materia de inversiones}

En la actualidad, los DESCA pretenden dejarse de lado por las implicaciones y obligaciones que requieren por parte de todos los integrantes de una sociedad. Por ello, las nuevas corrientes de pensamiento constitucional proponen diferentes soluciones para contrarrestar los grandes problemas a los cuales se ve enfrentado el postulado social dentro del modelo actual de Estado. Particularmente, se quiere analizar la teoría del ius constitutionale commune en América Latina (ICCAL), desarrollada en el Max Planck de Derecho Público Comparado y de Derecho Internacional, ${ }^{34}$ por cuanto intenta concretar estos principios que se encuentran en auge. La interacción de cortes, como se verá, es uno de los pilares en los cuales se sustenta esta nueva forma de ver el derecho constitucional. Nos detendremos en este punto, por considerar que se debe hacer extensivo a los tribunales de arbitramento que se encargan de fallar asuntos referidos a tratados de libre comercio o bilaterales de protección de inversiones, que pueden afectar aspectos sociales y el desarrollo económico de un país.

Antes de entrar en el punto mencionado anteriormente, es necesario entender así sea de manera instrumental en qué consiste el ICCAL, ${ }^{35}$ para posteriormente desarrollar las líneas de pensamiento que se proponen. El ICCAL, nace como una respuesta a los desafíos que el derecho constitucional y la teoría del Estado enfrentan en la actualidad; es decir, viene

\footnotetext{
34 Sobre el concepto ius constitutionale commune en América latina ver ibíd.; Armin von Bogdandy et al., Ius constitutionale commune en derechos humanos en América Latina, (México: Porrúa, 2013).

35 Véase en extenso Morales Antoniazzi, "El nuevo paradigma de la apertura de los órdenes...; José María Serna de la Garza, «Soberanía y apertura del Estado: una perspectiva mexicana», en Soberania y Estado Abierto en América Latina y Europa editado por Armin von Bogdandy et al., (México: Instituto de Investigaciones Jurídicas, 2014).
} 
a hacer frente a la situación de cesión de soberanía que otrora detentaba el Estado, como una prerrogativa con la cual podía establecer normas jurídicas y hacerlas cumplir a fin de poder materializar los postulados constitucionales. En ese sentido, era el poder supremo ante el cual un ciudadano se veía enfrentado. Hoy en día, los fenómenos económicos, sociales y culturales como la globalización, las inmigraciones y las pandemias, entre otros, han llevado a que este elemento del Estado se ceda en favor de otras instituciones internacionales y supranacionales, ${ }^{36}$ razón por la cual la concepción clásica de Estado se empezó a transformar.

Así pues, se encuentra que con el ICCAL se propone:

a) avanzar en el respeto de los principios de los derechos humanos, del Estado de derecho, y de la democracia; b) desarrollar el Estado abierto, y c) construir instituciones internacionales eficaces y legítimas. Los conceptos claves son 1) diálogo, 2) inclusión y 3) pluralismo normativo ${ }^{37}$.

i) Avanzar en el respeto de los principios de los derechos humanos, el Estado de Derecho y la democracia. Según los doctrinantes que desarrollan esta teoría se parte del punto de que el espacio estatal resulta insuficiente para poder darle la primacía a estos principios. En este sentido, sin restarle la importancia que tiene el ámbito estatal, se reconocen otras instituciones supranacionales que coadyuvan a la garantía de los derechos de las personas, la legalidad y los principios democráticos ${ }^{38}$.

ii) Estado abierto. Hace referencia a la apertura que tiene el Estado en materia normativa e instituciones públicas, es decir, que exista una oportunidad para adoptar en el ordenamiento jurídico interno fórmulas provenientes del derecho internacional y del derecho comparado que logren la garantía de los derechos

\footnotetext{
36 Sergio López-Ayllón, "The Impact of Globalization on the Reform of the State and Law in Latin America", Houston Journal of International Law 19, 3 (1997): 762; Serna de la Garza, "Soberanía y apertura del Estado...".

37 Armin von Bogdandy, "Ius constitutionale commune Latinoamericanum: una aclaración conceptual”, en Ius constitutionale commune en América Latina: rasgos, potencialidades y desafíos editado por Armin von Bogdandy et al., (México: Centro de Investigaciones Jurídicas, 2014), 9.
}

38 Ibíd. 
humanos. De igual forma, se hace especial énfasis en la adopción de los tratados internacionales, en especial los de derechos humanos. ${ }^{39}$

iii) Instituciones internacionales fuertes y legitimas. En primer lugar, al interior de las instituciones internacionales se debe generar un sistema consolidado de derechos humanos. En segundo, se requiere de instituciones que estén al tanto de los problemas actuales que permitan el fortalecimiento del Estado de derecho en el ámbito internacional y estatal al igual que los principios democráticos. ${ }^{40}$

Según von Bogdandy, ${ }^{41}$ estos tres principios del ICCAL vienen a articularse y cobrar de cierta manera una realización con la conjugación de tres conceptos que denomina clave, a saber:

i) Diálogo. En este punto se hace referencia a la interacción que debe existir entre tribunales. Los doctrinantes sostienen que en materia de decisiones judiciales no hay una última palabra; von Bogdandy manifiesta:

La decisión de un tribunal nacional de un país latinoamericano, aunque se trate de la Corte Suprema, puede ser controlado por la Corte Interamericana de Derechos Humanos. Al mismo tiempo, una decisión de la Corte Interamericana puede ser rechazada por un tribunal nacional..$^{42}$

Entonces, se dice en principio que existiría un constante diálogo a partir de las decisiones de las cortes a fin de generar mejores decisiones motivadas y más acordes a las garantías de los derechos de las personas en determinado Estado - más adelante se verán algunos comentarios que tenemos al respecto, ya que este elemento de la teoría es importante para este estudio-.

\footnotetext{
39 Ibíd.

40 Ibíd.

41 Ibíd.

42 Ibíd., 14.
} 
ii) Inclusión. Este concepto hace referencia a la lucha contra la exclusión y se impone según se entiende en este texto como una materialización del postulado social y las obligaciones que los poderes públicos tienen para su satisfacción.

iii) Pluralismo jurídico. Hoy en día, las teorías del monismo y el pluralismo resultan insatisfactorias, ya que no dan respuesta a fenómenos como la globalización. Así, la concepción que presenta esta teoría es

no considerar a las distintas normas del derecho internacional público, del derecho supranacional o del derecho estatal nacional, como parte de un ordenamiento jurídico unitario; por tanto, rechazan el paradigma de jerarquía como mecanismo que define la relación entre dichos ordenamientos. ${ }^{43}$

De esta manera los diferentes ordenamientos jurídicos entran a coadyuvar en la garantía de la dignidad de la persona.

Revisados de manera general los pilares sobre los cuales se estructura el ICCAL, se evidencia que lo que se pretende es dar relevancia a las cortes nacionales e internacionales, situándolas como una herramienta de instrumentalización y de reforzamiento del modelo de Estado. Pese a lo anterior, no hay que dejar de lado que las cortes a las que se hace referencia provienen de sistemas diferentes.

En este sentido, Serna de la Garza ${ }^{44}$ afirma que el sistema constitucional nacional y el derecho internacional de los derechos humanos son "dos regímenes paralelos de derecho positivo, dos esferas jurídiconormativas válidas en sus propios términos, en principio separadas, pero a la vez unidas por diversos puentes". ${ }^{45}$ Uno de esos puntos en común es la dignidad de la persona, la cual se impone como un principio fundador que da origen al ordenamiento jurídico y a las instituciones. ${ }^{46}$

\footnotetext{
43 Ibíd., 20.

44 José María Serna de la Garza, Impacto e implicaciones constitucionales de la globalización en el sistema jurídico mexicano, (México: UNAM, 2012).

45 Ibíd., pp. 243.

46 Francisco Fernández Segado, "La dignidad de la persona como fundamento de sus derechos", Revista de Derecho Público, 6 (1996); Héctor Gros Espiel, "La dignidad
} 
Pese a las diferencias y a las posibles contradicciones que puedan existir entre estos dos sistemas, Garza de la Serna realiza un estudio completo de cómo estos sistemas pueden entrar a conjugarse desde una visión del sistema regional latinoamericano. Examina cómo desde el control de constitucionalidad y convencionalidad se puede empezar a generar un diálogo entre cortes que permita tener un sistema armónico de protección de los derechos de las personas y tener al individuo como centro de atención del ordenamiento jurídico nacional e internacional. Finalmente, propone una serie de teorías que pueden ser adoptadas por las cortes nacionales e internacionales a fin de generar esa relación entre las cortes, esencial para el ICCAL. ${ }^{47}$

Ahora bien, queda claro que en la actualidad hay avances respecto de la necesidad que existe de generar un diálogo entre las cortes nacionales y las cortes internacionales de derechos humanos. De esta manera, se encuentra jurisprudencia y doctrina que trata de dar soluciones al respecto. ${ }^{48}$ La pregunta que surge en este punto es si existe un verdadero dialogo entre todas las cortes constitucionales nacionales e internacionales al momento de tomar sus decisiones.

Se puede partir diciendo que, en nuestros días, instituciones como la CIDH y las cortes nacionales que se encargan de salvaguardar la constitución han tenido un cambio en la forma en que se relacionan. Al respecto Góngora Mera ${ }^{49}$ sostiene que en la actualidad la estructura de justiciabilidad de los derechos humanos ha sufrido un cambio, que ha permitido generar "interacciones e influencias recíprocas" entre las cortes internacionales y nacionales. De este modo, se podría suponer, y en principio dar por sentado, que eventualmente existe un diálogo entre este

humana en los instrumentos internacionales sobre derechos humanos", Nueva Época 4, (2003): 205.

47 Serna de la Garza, Impacto e implicaciones...

Las teorías: (i) de la subsidiariedad; ii) la del margen de apreciación, y iii) interpretación conforme y principio pro homine.

48 Respecto del dialogo entre la Corte Interamericana de Derechos Humanos y la Corte Constitucional de Colombia véase, Manuel Góngora Mera, "Diálogos jurisprudenciales entre la corte interamericana de derechos humanos y la corte constitucional de Colombia: una visión coevolutiva de la convergencia de estándares sobre derechos de las víctimas", en La justicia constitucionaly su internacionalización editado por Armin von Bogdandy et al., (México: Universidad Autónoma de México, 2010).

49 Ibíd. 
tipo de cortes. En lo que se refiere a este escrito, la inquietud va dirigida particularmente a la relación entre las instancias jurisdiccionales encargadas de resolver los casos referidos a la solución de disputas generadas en virtud de los tratados bilaterales de protección de inversiones o de los tratados de libre comercio. ${ }^{50}$

Del anterior planteamiento se presentan al menos dos preguntas:

i) ¿Es posible subordinar los tribunales de arbitramento a la observancia y protección de los DESCA?

ii) ¿Debería existir una relación de diálogo entre los tribunales de arbitramiento y las cortes internacionales y nacionales encargadas de la protección de los derechos humanos y particularmente los DESCA como el propuesto por el ICCAL?

Respecto al primer cuestionamiento se puede aducir, en principio, que el problema de la sujeción y armonía entre dos regímenes, pertenecientes al ámbito internacional, surge de la fragmentación. De acuerdo con un informe presentado por la Comisión de Derecho Internacional, ${ }^{51} \mathrm{el}$ derecho internacional ha aumentado sus campos de acción de una manera vertiginosa. Esto ha generado una serie de consecuencias sucesivas que han permitido dar génesis al fraccionamiento del derecho internacional. Por esto se evidencia claramente el enfrentamiento que se puede generar entre el derecho internacional de inversiones y el derecho internacional de los derechos humanos.

La primera consecuencia que se presenta en el informe de la Comisión de Derecho Internacional es que el desarrollo del derecho internacional se ha producido de manera no coordinada. La dinámica de esta rama del derecho ha entrado a dar solución a aspectos específicos o

50 Respecto a los tratados bilaterales de protección de inversiones véase: Enrique Fernández Masía, "Arbitraje inversor-Estado: de "bella durmiente" a "león en la jungla"”, Revista Electrónica de Estudios Internacionales, 26 (2013): 4; José Carlos Fernández Rozas, "América Latina y el arbitraje de inversiones: ¿matrimonio de amor o matrimonio de conveniencia?", Revista de la Corte Española de Arbitraje, 24 (2009): 13-37; Stephan Schill, "International Investment Law and Public Comparative Law in Latin American Perspective", en International investment Law in latin america:problems and prospects Atila Tanzi et al., (Holanda: Nijhoff International Investment Law Series, 2016).

51 Comisión de Derecho internacional, Fragmentación del derecho internacional: dificultades derivadas de la diversificación y la expansión del derecho internacional, 2006. 
puntuales, sin detenerse a mirar la posibilidad de generar una legislación unificada que permita armonía. Al haber esferas normativas diferentes y en ocasiones totalmente apartadas, surgiría una segunda consecuencia que es la contradicción jurisprudencial. Estas contradicciones de las que habla la Comisión incluso se ha considerado que se dan dentro de las instituciones de arbitraje, como el CIADI. ${ }^{52}$ Esto perjudica a la seguridad jurídica y en consecuencia al rule of law. Al respecto, la Comisión sostuvo:

La fragmentación crea el peligro de que surjan normas, principios, sistemas de normas y prácticas institucionales contradictorios e incompatibles ${ }^{53}(. .$.$) Por ejemplo, el «derecho mercantil» y el «derecho$ ambiental» tienen objetivos sumamente específicos y se basan en principios que frecuentemente pueden apuntar a direcciones diferentes. ${ }^{54}$

De otro lado, algunos dirán que esta situación se predica en el derecho interno. Cabe recordar que dentro de los sistemas constitucionales occidentales existen una serie de normas que sirven como marco y límite al resto del ordenamiento jurídico, esto es la Constitución. Se debe manifestar que esta norma tiene como objeto primordial la dignidad de la persona, que es el cimiento de los derechos fundamentales y del ordenamiento jurídico en general. ${ }^{55}$ Esto repercute en que cada rama del derecho tendrá una regulación específica, siempre sometida a las normas constitucionales.

Siguiendo con este punto, se diría entonces que al existir un quebrantamiento dentro del derecho internacional no sería posible subordinar

52 Véase, William Burke-White y Andreas von Staden, "Private Litigation in a Public Law Sphere: The Standard of Review in Investor-State Arbitration", Yale Journal of International Law 35 (2010): 283-346. "Despite the growing number of arbitrations that have clear public law elements, ICSID tribunals have, as yet, failed to develop a coherent jurisprudential approach or consistent standard of review. Instead, ICSID arbitrations have generated a contradictory jurisprudence that lacks theoretical coherence..." (p. 297).

53 En lo referente a esta triple fragmentación puede verse: Gustavo Prieto Muñoz, "The EU as a Global Actor in the Post-Lisbon Era: Policy on International Investment and a Tale of Triple Fragmentation", en La politica de la Unión Europea en materia de derecho de las inversiones editado por Katia Fach Gómez, (Madrid: Bose, 2017), 25-44.

54 Comisión de Derecho Internacional, Fragmentación del derecho...

55 Francisco Fernández Segado, Estudios jurídico constitucionales, (México: Universidad Autónoma de México, 2003). 
los tribunales de arbitramento de inversiones. Sobre este aspecto y a fin de rebatir la afirmación anterior diremos que, en primer lugar, hay que remitirse a la Convención de Viena sobre los Tratados, particularmente al artículo 31 de este cuerpo normativo. Se sabe que la forma de interpretación sistemática de los tratados permitiría obligar a los árbitros a proferir sus fallos conforme a los parámetros de los derechos humanos. En virtud de este método de interpretación, los tratados internacionales de derecho público no deben ser analizados de manera separada y aislada; por el contrario, deberán tener en cuenta las demás normas que eventualmente tengan que ver con el asunto a resolver. En este sentido, las normas que hacen parte de un asunto de inversiones no serán exclusivamente las referidas a los procedimientos arbitrales que se caracterizan por ser normas que se encuentran bastante distanciadas con la protección de los derechos humanos, ya que su fuente de inspiración es el derecho mercantil.

Siguiendo con este punto, el CIADI en el fallo Urbaser S. A y Consorcio de aguas Bilbao Bi₹̨kaia, Bilbao Biskaia Ur Partzuergoa c. Argentina (CIADI no. ARB/07/26) intentó abrir la puerta para que los laudos arbitrales referidos a una inversión producto de un TBPI (tratado bilateral de protección de inversiones) sean resueltos teniendo como sustento jurídico normas de derecho internacional público, particularmente referentes a derechos humanos. Así, en principio recuerda que las empresas inversoras no pueden escudarse en que no son sujetos de derecho público internacional para eludir su obligación de sometimiento a los derechos humanos. A causa de esto trae a colación la Declaración Universal de Derechos Humanos (artículos 1, 21.2, 25.1 y 30), el Pacto Internacional de Derechos Económicos, Sociales y Culturales (artículos 11.1 y 5.1), la Declaración Tripartita de Principios sobre las Empresas Multinacionales, la Política Social de la Organización Internacional del Trabajo y la Convención de Viena (artículo 31.3) para demostrar que hoy en día no solo los Estados, sino también la sociedad civil y el mercado (entiéndase empresas) son parte esencial en la consolidación de los derechos humanos, por lo que su actuar en contra puede acarrear responsabilidad.

Sin embargo, cabe acotar que el fallo en mención posteriormente matizó su argumentación arguyendo que el deber de protección de los derechos humanos no es del todo imputable a las multinacionales. Por el contrario, sostiene:

si bien es correcto afirmar que la obligación del Estado se fundamenta en su obligación de hacer valer el derecho humano (...) las personas 
dentro de su jurisdicción, ello no es así en el caso de los inversores que persiguen, es cierto, el mismo objetivo, sino que se fundamenta en la Concesión y no en una obligación derivada del derecho humano al agua. De hecho, garantizar el derecho humano al agua representa una obligación de hacer. Esa obligación recae en los Estados y no es posible imponerla a una empresa (...) Para que esa obligación de hacer sea aplicable a un inversor particular, es necesario un contrato o una relación jurídica similar de derecho civil y comercial. (...) La situación es diferente si lo que está en juego es una obligación de no hacer, como la prohibición de realizar actos que violan los derechos humanos. Dicha obligación puede ser de aplicación inmediata no solo respecto de los Estados sino también respecto de las personas físicas y otros particulares. ${ }^{56}$

De lo anterior, se evidencia entonces que la posición del CIADI no pretende someterse del todo a los derechos humanos. Por el contrario, debe existir un contrato o documento jurídico que obligue al inversor a garantizar los derechos humanos refiriéndose a las obligaciones de hacer, ya que es un deber del Estado principalmente garantizar los derechos de sus habitantes. Sobre este punto refiere Echaide que es evidente que este tipo de tribunales no vinculen directamente la jurisprudencia en derechos humanos, debido a que en principio el asunto de las litis se refiere a asuntos que no conciernen directamente a este tipo de derechos.

Ahora bien, es erróneo pensar que el tema de los derechos humanos, en especial los desca, que son prestados también por inversores extranjeros (salud, educación, trabajo, servicios públicos), dependan de un acuerdo entre dos partes. De este modo, Echaide ${ }^{57}$ pone de manifiesto que el ius cogens sería aplicable de manera ineludible a los Estados en virtud de los artículos 53 y 64 de la Convención de Viena. Además, cree que para quienes consideren que estas normas no son para todos de obligatorio cumplimiento, se puede acudir a las obligaciones erga omnes ${ }^{58}$

\footnotetext{
56 Centro Internacional de Arreglo de Diferencias Relativas a Inversiones, Urbaser S.A y Consorcio de aguas Bilbao Bizkaia, Bilbao Biskaia Ur Partzuergoa c. Argentina, 2007, par. 1210.

57 Javier Echaide, "Demandas en el CIADI y el derecho humano al agua: ¿Tratados de inversiones vs. derechos humanos?”, Revista Colombiana de Derecho Internacional 15, 31 (2017): 81-114. https:/doi.org/10.11144/Javeriana.il14-31.dcdh

58 Las obligaciones erga omnes fueron estudiadas de manera breve, en un principio, en el fallo que la Corte Internacional de Justicia profirió en el asunto Barcelona Traction, Light
} 
que vincularían a toda la comunidad internacional. ${ }^{59}$ Por lo tanto, los tribunales de arbitramento deberían someterse a las normas que protegen los derechos humanos.

De hecho, ante la creciente importancia que tienen los derechos humanos, estos se deben de imponer sobre el ordenamiento en general ya que no se debe perder de vista que son estos los que sirven de pilar a la dignidad del hombre. En este mismo sentido, no resultaría raro que dentro de un arbitraje las partes hagan alusión al régimen de derecho internacional público. Esta idea no resulta del todo irracional ya que, si por ejemplo se revisa el Convenio de Washington de 1965, que constituyó el CIADI, su artículo 42 estipula que ante la falta de acuerdo respecto de las normas aplicables se podrán emplear "la legislación del Estado que sea parte en la diferencia, incluyendo sus normas de derecho internacional privado, y aquellas normas de derecho internacional que pudieren ser aplicables". En este sentido no es extraño que los árbitros tomasen normas ya sea del derecho interno de un país o normas internacionales que protejan los derechos de los individuos. ${ }^{60}$ Es pertinente resaltar que al momento de proferir un laudo arbitral en materia de inversiones es necesario realizar

and Power Company el 5 de febrero de 1970. En este documento la CIJ sostuvo: "When a State admits into its territory foreign investments or foreign nationals, whether natural or juristic persons, it is bound to extend to them the protection of the law and assumes obligations concerning the treatment to be afforded them. These obligations, however, are neither absolute nor unqualified. (...) In view of the importance of the rights involved, al1 States can be held to have a legal interest in their protection; they are obligations erga omnes. Such obligations derive, for example, in contemporary international law, from the outlawing of acts of aggression, and of genocide, as also from the principles and rules concerning the basic rights of the human person, including protection from slavery and racial discrimination. Some of the corresponding rights of protection have entered into the body of general international law (Reservations to the Convention on the Prevention and Punishment of the Crime of Genocide, Advisory Opinion, I.C.J. Reports 1951, p. 23); others are conferred by international instruments of a universal or quasi-universal character."

59 Ernst Petersmann, "Introduction and Summary: Administration of Justice in International Investment Law and Adjudication," en Human Rights in International Investment Law and Arbitration editado por Pierre-Marie Dupuy, (Oxford: Oxford University Press, 2009), 3-42.

60 Pierre-Marie Dupuy, "Unification rather fragmentation of international law? The case of international investment and human rights law, en Human Rights in International Investment Law and Arbitration Marie-Pierre Dupuy, (Oxford, Oxford University Press., 2009), 45-62. 
un análisis multidisciplinar que permitirá a posteriori generar una mejor decisión, cuyo enfoque no solo se centre en un aspecto económico. Sobre este aspecto Schill ${ }^{61}$ considera que dentro de las decisiones de los árbitros también está implícito el análisis del ejercicio del poder y la regulación de los Estados y de los derechos de los ciudadanos. ${ }^{62}$ Por ello se debe tener en cuenta la naturaleza del Estado que tiene implícita una serie de prerrogativas diferentes a las privadas.

Para resolver el segundo interrogante se debería empezar por mencionar que los árbitros con sus laudos tienen el poder de influenciar en la política económica y social de un Estado. Las disputas que se resuelven no se refieren de manera directa al cambio de la constitución, pero se debe advertir que existe la posibilidad de afectar la materialización e instrumentalización del Estado social y democrático de derecho. ${ }^{63}$ De igual forma en la relación que da génesis a los tribunales de arbitramento en materia de inversiones, a diferencia del arbitraje comercial, están presentes Estados. De esta forma, cabe pensar que solo por este hecho el arbitraje de inversiones debiese diferir del comercial y estar en mayor armonía con los derechos humanos. De cualquier modo, parte de la doctrina es consciente con que en los tratados de inversión se emplea el régimen privado a un ámbito netamente público, ya que entra a revisar las decisiones tomadas por el Gobierno de un Estado. ${ }^{64}$

Pese a lo mencionado anteriormente, van Harten y Martin Loughlin ${ }^{65}$ sostienen que a pesar de la atmosfera de derecho privado que envuelve la solución de controversias provenientes de un TBPI no hay que perder de vista que los árbitros son quienes van a controlar el ejercicio de las autoridades públicas, particularmente el quehacer del Gobierno. En consecuencia, al tener tal responsabilidad podría decirse que los trabajos de los centros de arbitraje dan lugar a lo que se llama el derecho

\footnotetext{
61 Schill, "International investment law and public...

62 Burke-White y von Staden, "Private Litigation in a Public Law Sphere...

63 "In these cases the need for public law standards of review is even more urgent as arbitral tribunals are transformed into public law, quasi-constitutional adjudicators" (Burke-White \& von Staden, 2010).

64 Ibíd.

65 Gus van Harten y Martin Loughlin, "Investment Arbitration as a Species of Global Administrative Law”, European Journal of International Law 17, no. 1 (2006): 121-150.
} 
administrativo global. ${ }^{66}$ Se sabe que el derecho administrativo domestico pretende racionalizar el poder del Estado a fin de que este se adecúe a las normas. ${ }^{67}$ De esta forma, cuando se acude a la jurisdicción contenciosoadministrativa o a los diversos mecanismos administrativos establecidos en la ley, lo que se pretende es controlar la organización administrativa y someterla a legalidad como principio rector sobre el cual deben fundar su quehacer el Gobierno y la administración. En vista de lo anterior, y si nos detenemos a ver la función de los tribunales de arbitramento, lo que se pretende es regular la actividad de la administración a partir de la legalidad y la imparcialidad en un asunto en el cual un particular (inversor extranjero) se siente afectado. ${ }^{68}$

De esta manera si se examina la concepción del régimen administrativo se encuentra que existen una serie de principios, como es el interés general o la dignidad de la persona, que son determinantes al momento de proferir una decisión pública o al instante en que un juez falle un litigio. Es por ello, que si se mira esta relación como un nuevo derecho administrativo de carácter global es necesario que los árbitros tengan en cuenta estos principios constitucionales. En el caso del derecho internacional encontramos que estos principios pueden ser perfectamente subsumidos por los tratados de derechos humanos. No se debe perder de vista que, al protegerse los derechos humanos, se garantizaría la dignidad de la

66 Respecto el derecho administrativo global véase: Benedict Kingsbury, Nico Krisch y Richard B. Stewart, "The Emergence of Global Administrative Law", Law and Contemporary Problems 68, 15 (2014): 15-61; Benedict Kingsbury y Stephan Schill, "Public Law Concepts to Balance Investors Rights with State Regulatory Actions in the Public Interest: The Concept of Proporcionality", en International Investment Law and Comparative Public Law editado por Stephan Schill, (Oxford, Oxford University Press, 2010), 76-104.

67 Ricardo Rivero Ortega, Derecho Administrativo, (Salamanca: Ratio Legis, 2016).

68 "Investment arbitration tribunals apply standards that constrain sovereign acts of a state's legislature, judiciary and administration. (...) Nonetheless, by obliging states to arbitrate disputes arising from sovereign acts, investment treaties establish investment arbitration as a mechanism to control the exercise of public authority. For this reason, in particular, investment arbitration is best analogized to domestic administrative law. In domestic administrative law, the primary subject of adjudicative review is executive government. In response to an individual claim, the courts may review sovereign acts of executive government to determine whether they were lawful and, if not, to adopt an appropriate remedy. In investment arbitration, by contrast, the main subject of adjudicative review is not executive government but the state as a whole" . Van Harten, y Loughlin, "Investment Arbitration as a Species of Global..., 146. 
persona, y en lo referente al interés general, de acuerdo con la teoría del doble carácter de los derechos fundamentales, los derechos de las personas obtienen una gran importancia dentro del quehacer administrativo y gubernamental. ${ }^{69}$

En este sentido es claro que debería existir un diálogo entre cortes ya que podemos ver que existe un punto en común que es el carácter público del Estado y su tarea fundamental de proteger los derechos de las personas. Así, al ser los tribunales de arbitramento entes que obtienen el carácter de autoridades encargadas de revisar la actuación del Estado, en el marco de una inversión, ${ }^{70}$ estos debiesen estudiar no solo las normas referentes a las establecidas por el tribunal UNCITRAL o las normas del CIADI. También deberían hacer, como sostiene Schill, un estudio comparado que permita poner en marcha esta nueva concepción de derecho administrativo global. ${ }^{71}$ De esta forma resultaría relevante tener en cuenta los pronunciamientos entre las cortes constitucionales nacionales del Estado huésped y las cortes de derechos humanos (TEDH o CIDH).

En aras de establecer un estándar de revisión en derecho público, es necesario que exista un diálogo entre los diferentes tribunales. Para ello se hace necesario que los árbitros al momento de pronunciarse cambien de mentalidad y dejen de verse como creadores de derecho netamente privado y por el contrario estén allende de esta posición. Ergo se requiere que sean capaces de ver que ocupan un rol determinante en el derecho público ya que como lo se mencionó anteriormente tienen un margen de decisión en la producción de las políticas públicas del Estado, en la regulación, en la garantía y satisfacción de los derechos de las personas y por supuesto en lo referente a los derechos del inversor. ${ }^{72}$

Respecto a este tema, Kingsbury y $\mathrm{Schill}^{73}$ sostienen que en lo que se refiere a la resolución de conflictos entre el inversor y otros intereses

\footnotetext{
69 Véase, Juan Felipe Solórzano Quintero, La asistencia jurídica pública al Estado y su infuencia en la actuación del poder Ejecutivo, (Salamanca: Universidad de Salamanca, 2016).

70 Armin von Bogdandy y Ingo Venzke, "Beyond Dispute: International Judicial Institutions as Lawmakers", German Law Journal 12, no. 5 (2011); Armin von Bogdandy y IngoVenzke, “¿En nombre de quién?”, en Diálogo jurisprudencial en derechos humanos: entre tribunales constitucionales y cortes internacionales editado por Eduardo Ferrer McGregor y Alfonso Herrera García, (Valencia, Tirant lo Blanch, 2013), 111.

71 Schill, "International Investment Law and Public Comparative...

72 Burke-White y von Staden, "Private Litigation in a Public Law Sphere...

73 Kingsbury y Schill, "Public Law Concepts to Balance Investors Rights..., 76.
} 
públicos se hace imperioso usar conceptos de derecho público empleados por las cortes internacionales y nacionales, especialmente recurriendo a un análisis de proporcionalidad ${ }^{74}$ "in order to balance rights and rightslimiting policy choices".

\section{Conclusiones}

Estamos en presencia de un desplazamiento del Estado por parte de fuerzas económicas que intentan en cierta forma cooptar los poderes públicos o simplemente pretenden yuxtaponer otras instituciones que se adecúen a sus necesidades. De esta forma, somos conscientes de que las grandes potencias y las multinacionales han dado génesis a un modelo neoliberal que pretende ser impuesto a través de la reforma de la constitución, las leyes o de superposición de las autoridades públicas. ${ }^{75}$

Esto ha generado una serie de inconsistencias en los sistemas jurídicos nacionales e internacionales que en ocasiones no pueden brindar la salvaguarda de los derechos de las personas, ya que las normas en esta materia resultan insuficientes e ineficaces. Sin embargo, existen aspectos que normativamente resultan paradójicamente eficaces y eficientes como la inversión extranjera, de esta manera se evidencia la gran fuerza con que cuentan las grandes potencias y multinacionales para imponer su voluntad.

No obstante, el mundo ha tratado de reaccionar y en la actualidad se generan teorías jurídicas que pretenden dar una respuesta a estas situaciones que afectan a muchos Estados, particularmente a los que económicamente se encuentran subyugados a otros o dependen económicamente de las inversiones. Una de estas respuestas la da el ICCAL que a partir de una visión transformadora del derecho constitucional pretende que el modelo de Estado tenga una mayor instrumentalización y se puedan generar cambios de verdadera protección y goce de los postulados sociales democráticos y de derecho.

\footnotetext{
74 Respecto de la proporcionalidad véase, ibíd.

75 Arminda Balbuena Cisneros et al., Estado de Derecho y crisis en América Latina: algunas notas entre la pesadilla y la esperanza, (Alicante, Universidad de Alicante, 2005); Calros de Cabo Martín, La crisis del Estado social, (Barcelona, Ppu, 1986); Gerardo Pisarello Prados, "Globalización, mundialización y crisis del concepto de soberanía", en Globalización mundialización y crisis del concepto de soberania: algunos efectos en América latina y en Europa editado por Gerardo Pisarello Prados y Antonio de Cabo de la Vega, (Alicante, Ediciones Universidad de Alicante, 2000).
} 
Para ello, se le da un particular enaltecimiento a los tribunales internacionales y nacionales a fin de que sean estos los que generen un cambio en la consolidación de todos los fines constitucionales de cada Estado. Por ello, son estos los que a partir de la verdadera imposición de justicia pueden garantizar en primer lugar el Estado de derecho y como consecuencia de esto dar paso a la materialización de los postulados democráticos y sociales. En este punto y para el logro de lo mencionado anteriormente se propone un diálogo entre cortes y tribunales tanto estatales como internacionales. Pero como se mencionó anteriormente existen intereses privados que no tienen dentro de sus prioridades las pretensiones sociales. De esta manera, se mostró que los árbitros encargados de impartir justicia en temas referentes a inversiones deben empezar a tener un sometimiento a los derechos humanos; además, se pueden establecer lazos de entre las cortes nacionales e internacionales para que en su conjunto instrumentalicen esas trasformaciones sociales que la sociedad necesita.

De igual forma, no se debe perder de vista que los tribunales de arbitramento son una instancia en la cual se juzga el quehacer del ejecutivo, por lo que la "actividad judicial" debe tener en cuenta la naturaleza del Estado y todo lo que esta persona jurídica implica para la satisfacción de los anhelos, mandatos y derechos que se necesitan para lograr el bienestar general de la sociedad. Por ello se considera que debemos adentrarnos en la concepción de un derecho administrativo global, que tenga en cuenta todo este componente propio del derecho público.

\section{Referencias}

\section{Libros y capítulos de libros}

Aguilar Cavallo, Gonzalo. “¿Son los derechos sociales sólo apreciaciones? Perspectivas de la Comisión Interamericana de Derechos Humanos", en Construcción y papel de los derechos sociales fundamentales editado por Armin von Bogdandy. México: Instituto de Investigaciones Jurídicas, 2011.

Alexy, Robert. Teoría de los derechos fundamentales. Madrid, Centro de Estudios Políticos y Constitucionales, 2007.

Balbuena Cisneros, Arminda et al. Estado de derecho y crisis en América Latina: algunas notas entre la pesadilla y la esperanza. Alicante, Universidad de Alicante, 2005.

Arango, Rodolfo. El concepto de los derechos. Bogotá: Legis, 2015. 
Sudáfrica, Corte Constitucional. Government of the Republic of South Africa. \& Ors v Grootboom \& Ors, 17 de diciembre de 1999.

Bernal Pulido, Carlos. "Capítulo introductorio". en Teoría de los derechos fundamentales editado por Roberts Alexy. Madrid, Centro de Estudios Políticos y Constitucionales, 2007.

Böckenförde, Wolfgang. Escritos sobre derecbos fundamentales. Baden-Baden: Nomos, 1993.

von Bogdandy, Armin. "La protección de los vulnerables: un ejemplo de gobernanza posnacional". En Construcción y papel de los derechos sociales fundamentales editado por Armin von Bogdandy et al., 313-37. México: Instituto de Investigaciones Jurídicas, 2011.

von Bogdandy, Armin \& Venzke, Ingo. “¿En nombre de quién?”. En Diálogo jurisprudencial en derechos humanos: entre tribunales constitucionales $y$ cortes internacionales editado por Eduardo Ferrer Mac-Gregor y Alfonso Herrera García, 83-130. Valencia: Tirant lo Blanch, 2013. Armin von Bogdandy et al., "Ius constitutionalle commune en América Latina: un enfoque regional del constitucionalismo transformador'. En Ius constitutionale commune en América Latina: textos básicos para su comprensión editado por Armin von Bogdandy et al., 17-54. Querétaro: Instituto de Estudios Constitucionales del Estado de Querétaro, 2017.

von Bogdandy, Armin. "Ius constitutionale commune Latinoamericanum: una aclaración conceptual". En Ius constitutionale commune en América Latina: textos básicos para su comprensión editado por Armin von Bogdandy et al., 17-54. Querétaro: Instituto de Estudios Constitucionales del Estado de Querétaro, 2017.

Bohoslavsky, Juan \& Bautista, Justo. "Compatibilizando derechos de los inversores extranjeros y derechos humanos: ¿Por Qué? ¿Cómo? ¿Quién? ¿Cuándo?”. En International Investment Law in Latin America: Problems and Prospects editado por Atilla Tanzi et al. Holanda, Nijhoff international investment law series, 2016.

Bohoslavsky, Juan \& Bautista, Justo. Protección del derecho humano al agua y arbitrajes de inversión. Santiago de Chile: CEPAL, 2011.

de Cabo Martín, Carlos. La crisis del Estado social. Barcelona: Ppu, 1986.

Carbonell, Miguel \& Ferrer Mac-Gregor, Eduardo. Los derechos sociales y su justiciabilidad directa. México: Universidad Autónoma de México, 2014.

Dupuy, Pierre-Marie. "Unification Rather Fragmentation of International Law? The Case of International Investment and Human Rights Law. En Human Rights in International Investment Law and Arbitration editado por Dupuy, Pierre-Marie. Oxford: Oxford University Press, 2009. 
Fabre, Cécile. Social Rights under the Constitution. Oxford: Oxford University Press, 2000.

Comisión de Derecho internacional. Fragmentación del derecho internacional: dificultades derivadas de la diversificación y la expansión del derecho internacional, 2006.

Fernández García, Eusebio. Dignidad humana y ciudadanía cosmopolita. Madrid: Dykinson, 2001.

Fernández Segado, Francisco. Estudios jurídico constitucionales. México, Universidad Autónoma de México, 2003.

Forsthoff, Ernst. "Concepto y esencia del Estado Social de Derecho". En El estado social, editado por Wolfgang Abendrot Ernst Forsthoff y Karl Doehring, 99-101. Madrid: Centro de Estudios Constitucionales, 1986.

Góngora Mera, Manuel. “Diálogos jurisprudenciales entre la corte interamericana de derechos humanos y la corte constitucional de Colombia: una visión coevolutiva de la convergencia de estándares sobre derechos de las víctimas". En La justicia constitucional y su internacionalización editdo por Armin von Bogdandy et al. México: Universidad Autónoma de México, 2010.

Hesse, Konrad. Grunzüge des Verfassungsrechts der Bundesrepublik Deutschland. Heidelberg: C.F. Müller, 1991.

Hesse, Konrad. "El significado de los derechos fundamentales". En Manual de derecho constitucional editado por Antonio López Pina. Madrid: Marcial Pons, 2001.

Kingsbury, Benedict \& Schill, Stephan. "Public Law Concepts to Balance Investors Rights with State Regulatory Actions in the Public Interest: The Concept of Proporcionality". En International Investment Law and Comparative Public Law editado por Stepahn Schill, 76-104. Oxford: Oxford University Press, 2010.

León Alonso, Marta. La protección constitucional de la salud en el Marco del Estado Social y Democrático de Derecho. Salamanca: Ediciones Universidad de Salamanca, 2009.

Parra Vera, Óscar. "La justiciabilidad de los derechos económicos sociales y culturales en el sistema interamericano a la luz del artículo 26 de la Convención Americana: El sentido y la promesa del caso Lagos del Campo". En Inclusión, ius commune y justiciabilidad de los desc en la jurisprudencia interamericana: el caso Lagos del Campo y los nuevos desafíos editado por Eduardo Ferrer Mac-Gregor et al., 181-234 
Querétaro: Instituto de estudios constitucionales del Estado de Querétaro, 2018.

Morales Antoniazzi, Mariela. "El nuevo paradigma de la apertura de los órdenes constitucionales: una perspectiva sudamericana". En Soberania y Estado abierto en América Latina y Europa editado por Armin von Bogdandy et al., 233-82. México: Instituto de Investigaciones Jurídicas.

Petersmann, Ernst. "Introduction and Summary: Administration of Justice in International Investment Law and Adjudication." En Human Rights in International Investment Law and Arbitration editado por Pierre-Marie Dupuy, 5 y ss. Oxford, Oxford University Press, 2009. Piovesan, Flavia. "Protección de los derechos sociales: reto de un ius commune para Sudamérica". En Construcción y papel de los derechos socialesfundamentales editado por Armin von Bogdandy et al., 339-80. México, Universidad Autónoma de México, 2011.

Pisarello Prados, Gerardo. "Los derechos sociales y sus 'enemigos': Elementos para una reconstrucción garantista". En Defendery repensar los derechos sociales en tiempo de crisis editado por Gerardo Pisarello Prados, 13-22. Barcelona: Observatorio desc, 2009.

Pisarello Prados, Gerardo. "Globalización, mundialización y crisis del concepto de soberanía". En Globalización mundialización y crisis del concepto de soberania: algunos efectos en América latina y en Europa editado por Gerardo Pisarello Prados y Antonio de Cabo de la Vega, 23-54. Alicante: Ediciones Universidad de Alicante, 2000.

Prieto Muñoz, Gustavo. "The eu as a Global Actor in the Post-Lisbon Era: Policy on International Investment and a Tale of Triple Fragmentation". En La politica de la Unión Europea en materia de derecho de las inversiones editado por Katia Fach Gómez, 25-44. Madrid: Bose, 2017.

Rivero Ortega, Ricardo. Derecho administrativo. Salamanca: Ratio Legis, 2016. Schill, Stephan. "International Investment Law and Public Comparative Law in Latin American Perspective". En International Investment Law in Latin America: Problems and Prospects editado por Atila Tanzi et al. Holanda: Nijhoff International Investment Law Series, 2016.

Serna de la Garza, José María. "Soberanía y apertura del Estado: una perspectiva mexicana". En Soberanía y Estado Abierto en América Latina y Europa editado por Armin von Bogdandy et al., 1-56. México: Instituto de investigaciones jurídicas, 2014. 
Serna de la Garza, José María. Globalización gobernanza: las transformaciones del Estado y sus implicaciones para el derecho público: contribución para una interpretación del caso guardería abc. México: UNAM, 2010.

Serna de la Garza, José María, Impacto e implicaciones constitucionales de la globalización en el sistema jurídico mexicano, México, UNAM, 2012.

Solano, Nancy \& Sepúlveda, Myriam. Metodología de la investigación social jurídica. Bogotá: Ibáñez, 2008.

Solórzano Quintero, Juan Felipe. La asistencia jurídica pública al Estado y su influencia en la actuación del poder Ejecutivo. Salamanca: Universidad de Salamanca, 2016.

Vogel, Klaus. Die Verfassungentscheidung des Grungesetzes für die internationale Zusammenarbeit. Tübingen: Mohr, 1964.

\section{Artículos}

von Bogdandy, Armin \& Venzke, Ingo. "Beyond Dispute: International Judicial Institutions as Lawmakers". German Law Journal 12, no. 5 (2011): 979-1003.

Burke-White, William \& von Staden, Andreas. "Private Litigation in a Public Law Sphere: The Standard of Review in Investor-State Arbitration". Yale Journal of International Law 35, no. 2 (2010): 283-346.

Echaide, Javier. "Demandas en el ciadi y el derecho humano al agua: $¿$ Tratados de inversiones vs. derechos humanos?”. Revista Colombiana de Derecho Internacional15, no. 31 (2017). https:/doi.org/10.11144/ Javeriana.il14-31.dcdh

Fernández Masía, Enrique: "Arbitraje inversor-Estado: de "bella durmiente" a "león en la jungla"". Revista Electrónica de Estudios Internacionales, no. 26 (2013): 1-27.

Fernández Segado, Francisco. "La dignidad de la persona como fundamento de sus derechos". Revista de Derecho Público, no. 6 (1996).

Fernández Rosas, José Carlos. "América Latina y el arbitraje de inversiones: ¿matrimonio de amor o matrimonio de conveniencia?”. Revista de la Corte Española de Arbitraje 24, (2009): 13-37.

Fix-Fierro, Héctor \& López-Ayllón, Sergio. "The Impact of Globalization on the Reform of the State and Law in Latin America". Houston Journal of International Law, no. 3 (1997): 785-801. Recuperado de http://www.hjil.org/articles/hjil-19-3-fierro-ayllon.pdf

Gros Espiel, Héctor. "La dignidad humana en los instrumentos internacionales sobre derechos humanos". Nueva Época 4, (2003): 193-223. 
van Harten, Gus \& Loughlin, Martin. "Investment Arbitration as a Species of Global Administrative Law". European Journal of International Law 17, no. 1 (2006): 121-150.

Kingsbury, Benedict, Krisch, Nico, \& Stewart, Richard B. "The Emergence of Global Administrative Law." Law and Contemporary Problems 68, (2014): 15-61.

Mathews, Jessica. "Power Shift". Foreign Affairs 76, (1997): 50-66.

\section{Jurisprudencia}

Centro Internacional de Arreglo de Diferencias Relativas a Inversiones, Urbaser S.A y Consorcio de aguas Bilbao Bizkaia, Bilbao Biskaia Ur Partzuergoa v. Argentina, 2007.

Corte Interamericana de Derechos Humanos, sentencia caso Suárez Peralta v. Ecuador, 21 de mayo de 2013.

Corte Interamericana de Derechos Humanos, sentencia caso Suárez Peralta v. Ecuador, 21 de mayo de 2013, en especial voto recurrente del juez Ferrer- Mac-Gregor.

Corte Interamericana de Derechos Humanos, sentencia caso Acevedo Buendía v. Perú, 1 de julio de 2009.

Corte Internacional de Justicia, sentencia Barcelona Traction, Light and Power Company el 5 de febrero de 1970.

Colombia, Corte Constitucional, sentencia C-587 de 1992, de 12 de noviembre.

Colombia, Corte Constitucional, sentencia T- 596 de 1992, diciembre 10.

España, Tribunal Constitucional STC 53/1985, de 11 de abril.

España, Tribunal Constitucional STC 25/1981, de 14 de julio. 